\title{
Fast Vesicle Recycling Supports Neurotransmission during Sustained Stimulation at Hippocampal Synapses
}

\author{
Yildirim Sara, Marina G. Mozhayeva, Xinran Liu, and Ege T. Kavalali \\ Center for Basic Neuroscience and Department of Physiology, University of Texas Southwestern Medical Center, Dallas, \\ Texas 75390-9111
}

\begin{abstract}
High-frequency induced short-term synaptic depression is a common feature of central synapses in which synaptic responses rapidly decrease to a sustained level. A limitation in the availability of release-ready vesicles is thought to be a major factor underlying this phenomenon. Here, we studied the kinetics of vesicle reavailability and reuse during synaptic depression at hippocampal synapses. High-intensity stimulation of neurotransmitter release was induced by hyperosmolarity, high potassium, or action potential firing at $30 \mathrm{~Hz}$ to produce synaptic depression. Under these conditions, synaptic transmission rapidly depressed to a plateau level that was typically $10-40 \%$ of the initial response and persisted at this level for at least 5 min regardless of the developmental stage of synapses. This nondeclining phase of transmission was partly sustained by fast recycling and reuse of synaptic vesicles even after
\end{abstract}

In the CNS, synapses are faced with significant challenges to sustain neurotransmission over a wide range of stimulation frequencies. Structural studies and functional analysis have shown that central synapses have a small number of functional synaptic vesicles, especially during early stages of maturation after synaptogenesis (Vaughn, 1989; Fiala et al., 1998; Mozhayeva et al., 2002). Restrictions on synaptic function imposed by the limited number of vesicles are partly compensated by recycling and reuse of synaptic vesicles that have undergone exocytosis (Südhof, 2000; Harata et al., 2001; Wilkinson and Cole, 2001). These limitations in synaptic vesicle supply are revealed during sustained highfrequency stimulation, in which most synapses exhibit extensive depression, measured as a marked decrease in the postsynaptic responses to a plateau level. A current model of this phenomenon suggests that the early phase of short-term synaptic depression results from depletion of vesicles in the readily releasable vesicle pool (RRP), presumably corresponding to vesicles juxtaposed to the active zone, coupled with a decrease in release probability. During the depressed plateau phase, synapses are thought to release vesicles that transiently populate the RRP from a reserve pool (Zucker, 1989; Stevens and Tsujimoto, 1995; Neher, 1998; $\mathrm{Wu}$ and Betz, 1998; Regehr and Stevens, 2001). The vesicles in the reserve pool are anatomically distant from release sites and

Received Oct. 24, 2001; revised Dec. 4, 2001; accepted Dec. 13, 2001.

E.T.K is the Effie Marie Cain endowed scholar in biomedical research at the University of Texas Southwestern Medical Center. We thank Kimberly Huber, Tuhin Virmani, and Thomas Südhof for helpful discussions and for critically reading this manuscript.

Correspondence should be addressed to Dr. Ege T. Kavalali, Center for Basic Neuroscience, University of Texas Southwestern Medical Center, 5323 Harry Hines Boulevard, Dallas, TX 75390-9111. E-mail: Ege.Kavalali@UTSouthwestern.edu. Copyright (C) 2002 Society for Neuroscience $0270-6474 / 02 / 221608-10 \$ 15.00 / 0$ minutes of stimulation. Simultaneous electrical recording of postsynaptic responses and styryl dye destaining showed that after an initial round of exocytosis, vesicles were available for reuse with a delay between 1 and $3 \mathrm{sec}$ during $30 \mathrm{~Hz}$ action potential or hypertonicity-induced stimulation. During these stimulation paradigms, there was a limited mobilization of vesicles from the reserve pool. During $10 \mathrm{~Hz}$ stimulation, however, the extent of vesicle reuse was minimal during the first $20 \mathrm{sec}$. These results suggest a role for fast vesicle recycling as a functional homeostatic mechanism that prevents vesicle depletion and maintains synaptic responses in the face of intense stimulation.

Key words: synaptic vesicle recycling; endocytosis; synaptic depression; FM1-43; patch clamp; hippocampal culture become functionally competent for release during stimulation and replace vesicles in the RRP. The RRP and the reserve pool together make up the recycling pool of vesicles, which correspond to all vesicles capable of activity-dependent recycling on stimulation.

In its current state, this linear model does not take into account the reuse of recycled synaptic vesicles during synaptic depression. This omission is supported by experiments conducted in neuromuscular junction and hippocampal synapses that estimated the time required for recycled synaptic vesicles to mix with the nonreleased population of vesicles and to rejoin neurotransmission to be between 40 and $90 \mathrm{sec}$ (Betz and Bewick, 1993; Ryan et al., 1993; Liu and Tsien, 1995). Recent studies that examine the recovery of synaptic responses in "pulse-chase" experiments after depletion of RRP by brief stimulation showed that RRP vesicles were reused within seconds (Pyle et al., 2000). However, the extent of vesicle reavailability and reuse under circumstances in which these mechanisms would be most advantageous, such as during intense stimulation or at early synaptic development, has not been determined. To determine the respective roles of fast vesicle reuse and vesicle replenishment from the reserve pool during synaptic depression, we monitored vesicle reavailability during depression induced by multiple forms of sustained stimulation at distinct stages of synaptic development. In particular, we set out to determine the correlation between synaptic transmission, which registers fusion events independent of the use history of vesicles, and destaining kinetics of styryl dye FM2-10, a onetime marker of vesicle fusion.

\section{MATERIALS AND METHODS}

Cell culture. CA3-dentate gyrus regions were dissected from hippocampi of 1- to 2-d-old Sprague Dawley rats, and dissociated cultures were 
prepared according to previously published protocols (Kavalali et al., 1999a).

Electron microscopy. The cells were fixed for $30 \mathrm{~min}$ in $2 \%$ glutaraldehyde buffered with $0.1 \mathrm{M}$ sodium phosphate, $\mathrm{pH} 7.2$ at $4^{\circ} \mathrm{C}$. They were rinsed twice in buffer and then incubated in $1 \% \mathrm{OsO}_{4}$ for $30 \mathrm{~min}$ at room temperature. After rinsing with distilled water, specimens were stained en bloc with $2 \%$ aqueous uranyl acetate for $15 \mathrm{~min}$, dehydrated in ethanol, and embedded in poly/bed 812 for $24 \mathrm{hr}$. Fifty-nanometer sections were poststained with uranyl acetate and lead citrate and viewed with a Philips CM-12 transmission electron microscope.

Fluorescence imaging. Synaptic boutons were loaded with FM2-10 (400 $\mu \mathrm{M}$; Molecular Probes, Eugene, OR) during a $90 \mathrm{sec}$ incubation in the hyperkalemic solution $45 \mathrm{mM} \mathrm{K}^{+} / 2 \mathrm{mM} \mathrm{Ca}^{2+}$. This protocol gives maximal labeling, as judged by comparison with loading with 1200 action potentials (APs) applied at $10 \mathrm{~Hz}$, which labels $90 \%$ of this total pool. A modified Tyrode solution used in all experiments contained (in mM): 150 $\mathrm{NaCl}, 4 \mathrm{KCl}, 2 \mathrm{MgCl}_{2}, 10$ glucose, $10 \mathrm{HEPES}$, and $2 \mathrm{CaCl}_{2}, \mathrm{pH} 7.4$ $(\sim 310 \mathrm{mOsm})$. A hypertonic solution was prepared by addition of 500 $\mathrm{mm}$ sucrose to the Tyrode solution, and high- $\mathrm{K}^{+}$solutions contained equimolar substitution of $\mathrm{KCl}$ for $\mathrm{NaCl}$. Field stimulation was applied through parallel platinum electrodes immersed into the perfusion chamber delivering $30 \mathrm{~mA} 1 \mathrm{msec}$ pulses. All staining and washing protocols were performed with $10 \mu \mathrm{M}$ CNQX and $50 \mu \mathrm{M}$ AP-5 to prevent recurrent activity. During simultaneous optical and electrophysiological recordings (see Figs. 5-8), transmission blockers were omitted from the bath. This omission did not give rise to significant alterations in destaining kinetics compared with previous work. Images were taken after 10 min washes in dye-free solution in nominal $\mathrm{Ca}^{2+}$ to minimize spontaneous dye loss. In all experiments, we selected isolated boutons $\left(\sim 1 \mu \mathrm{m}^{2}\right)$ for analysis and avoided apparent synaptic clusters (Kavalali et al., 1999a). Destaining of hippocampal terminals with a hypertonic, high-potassium challenge was achieved by direct perfusion of solutions onto the field of interest by gravity $(2 \mathrm{ml} / \mathrm{min})$. In experiments in which FM2-10-containing solution was washed out within seconds (see Fig. 4), we increased the perfusion rate to $10 \mathrm{ml} / \mathrm{min}$. Adjustment of the hyperosmotic solution flow rate was critical to prevent alterations in fluid levels and fluorescence values during rapid solution exchanges. Fluorescence values were not significantly distorted by cell shrinkage during sucrose application. Images were obtained by a cooled, intensified digital CCD camera (Roper Scientific, Trenton, NJ) during illumination (1 Hz and $40 \mathrm{msec})$ at $480 \pm$ $20 \mathrm{~nm}$ (505 dichroic longpass and $535 \pm 25$ bandpass) via an optical switch (Sutter Instruments, Novato, CA). Images were acquired and analyzed using Axon Imaging Workbench software (Axon Instruments, Union City, CA).

Electrophysiology. Synaptic responses were recorded from pyramidal cells using a whole-cell configuration of the patch-clamp technique. Data were acquired using an Axopatch 200B amplifier and Clampex 8.0 software (Axon Instruments). Recordings were filtered at $2 \mathrm{kHz}$ and sampled at $200 \mu \mathrm{sec}$. The pipette internal solution included (in $\mathrm{mM}$ ): 115 Cs-MeSO $3,10 \mathrm{CsCl}, 5 \mathrm{NaCl}, 10$ HEPES, 0.6 EGTA, 20 TEA-Cl, 4 Mg-ATP, $0.3 \mathrm{Na}_{3} \mathrm{GTP}$, and 10 lidocaine $N$-ethyl bromide, $\mathrm{pH} 7.35$ (300 mOsm). Picospritzer-delivered pulses of hypertonic sucrose $(+500$ mOsm) were applied to proximal dendrites. For AP-dependent stimulation, we used the same technique as in imaging experiments. Error bars denote SEM.

\section{RESULTS}

\section{Steady-state level of neurotransmission during prolonged stimulation}

Maturation of presynaptic morphology is associated with an increase in the number of synaptic vesicles and a delayed emergence of a sizable reserve pool. The delayed emergence of vesicle pool hierarchy during development would be expected to have a large impact on the regulation of synaptic responses to sustained high-frequency stimulation (Mozhayeva and Kavalali, 2000). At early stages of maturation, synapses with few vesicles, of which a large fraction is in a functionally docked state, should manifest significant synaptic depression during high-frequency stimulation. Mature synapses, on the other hand, possess a large number of vesicles and a substantial reserve pool and thus should be able to sustain neurotransmission for longer periods. To test these predictions, we characterized the time course and amount of synaptic
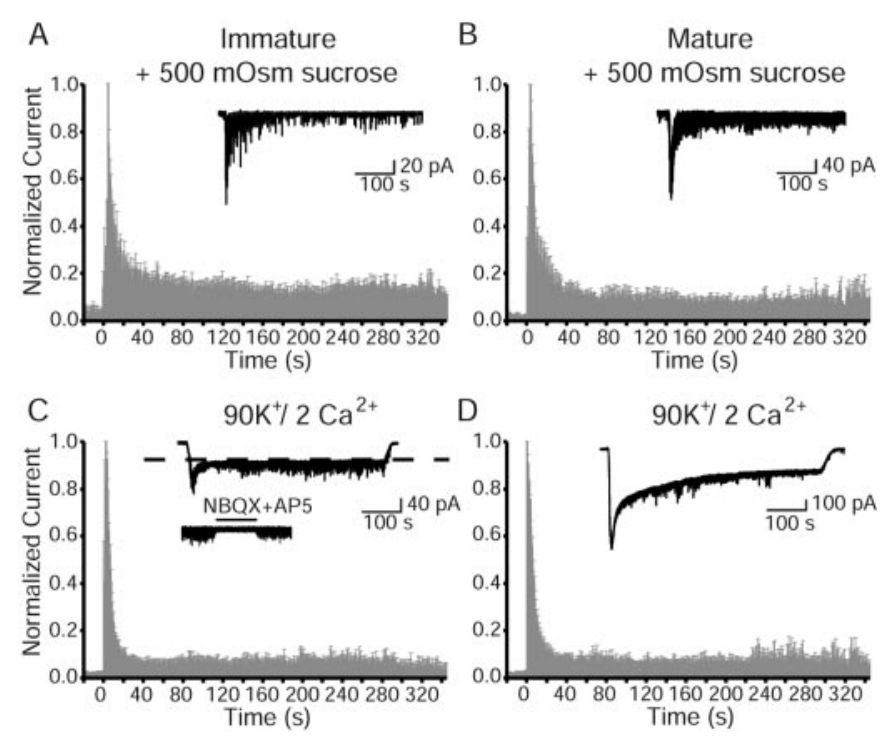

Figure 1. Prolonged high-potassium or hypertonic sucrose solution application gives rise to sustained neurotransmission throughout synapse maturation. $A, B$, During whole-cell recordings introduction of hypertonic $(+500 \mathrm{mOsm})$ solution initiated transmitter release with a peak response followed by a prominent plateau in immature (6-7 div) and mature (14-16 div) cultures $(n=6$ each). Plots show average normalized current transfer integrated over $1 \mathrm{sec}$ intervals. Example traces are depicted in the insets. $C, D, \mathrm{~K}^{+}(90 \mathrm{~mm})$ stimulation caused rapid depolarization and synaptic activity $(n=6)$. We isolated the amount of synaptic activity from the nonsynaptic current influx by subtracting the baseline current after blockade of postsynaptic receptors by 2,3-dihydroxy-6-nitro-7-sulfonylbenzo[f]quinoxaline $(N B Q X)(10 \mu \mathrm{M})$ and AP-5 $(50 \mu \mathrm{M})$ during a subsequent application of high- $\mathrm{K}^{+}$solution with the same duration. This baseline typically corresponds to the level indicated by the dashed line in $C$. The effect of glutamate receptor inhibitors during plateau is shown in the inset. All symbols show mean values \pm SEM.

depression induced by prolonged ( $>5 \mathrm{~min}$ ) applications of hypertonic sucrose $(+500 \mathrm{mOsm})$ or high-potassium stimulation (90 $\mathrm{mM} \mathrm{K}{ }^{+} / 2 \mathrm{mM} \mathrm{Ca}^{2+}$; Fig. 1). The $5 \mathrm{~min}$ time frame provides an ample period for vesicle depletion and use of the total recycling pool, primarily because this duration is at least three times longer than the previous estimates of vesicle exchange and mixing between the RRP and the reserve pool (Murthy and Stevens, 1999; Pyle et al., 2000). Surprisingly, both immature [6-7 d in vitro (div); $n=6$ ] and mature ( $>15$ div; $n=6)$ synapses responded robustly to these stimulation paradigms and showed a significant plateau phase of neurotransmitter release after initial synaptic depression. Although the size of the total recycling pool dramatically increases between these two stages of synaptic development (Mozhayeva and Kavalali, 2000), there were no major quantitative differences in the time course of synaptic depression and the level of plateau reached. Most surprisingly, in the case of hypertonic sucrose stimulation, a rapid rise and decline in neurotransmitter release was followed by a prominent plateau phase of activity at $\sim 20 \%$ of the peak for both immature and mature synapses (Fig. 1A,B). This sucrose-evoked neurotransmitter release was vesicular in nature, because in experiments at 6 div in which a small number of synapses are stimulated, we could clearly identify individual quantal events even after $300 \mathrm{sec}$ of stimulation (data not shown). The minutes-long enhancement of baseline activity was in striking contrast to what is generally thought to be a transient effect of hyperosmotic shock. A transient mechanical action on synaptic junctions may explain release of docked vesi- 
cles as well as replenishment of recently vacated docking sites from a reserve pool; however, it is difficult to account for the minutes-long sustained activity without invoking a form of vesicle recycling operating under these conditions.

To corroborate the argument that hypertonic sucrose application causes a form of continuous vesicle recycling, we performed imaging experiments in which we applied +500 mOsm solution for 5 min to synapses loaded with FM2-10, a fast departitioning dye, during $90 \mathrm{sec}$ application of $45 \mathrm{~mm} \mathrm{~K}^{+} / 2 \mathrm{mM} \mathrm{Ca}^{2+}$ solution. Within $15 \mathrm{sec}$ of sucrose application, we could destain $16.6 \pm$ $0.1 \%$ of the total fluorescence ( $n=3 ; 221$ boutons), whereas at the end of the stimulation, $245 \mathrm{sec}$ later, the destaining reached $35.9 \pm 0.4 \%$ of the total. On average, $65 \%$ of the initial FM2-10 fluorescence still remained within the boutons by the end of $5 \mathrm{~min}$ of sucrose stimulation. Limited destaining achieved by sucrose application was in contrast to the sustained level of synaptic activity that could be induced by this stimulation. These observations support the argument that the action of hypertonic sucrose stimulation is limited to a subset of vesicles that correspond to the readily releasable pool. We also repeated the same electrophysiological and optical protocols on synapses pretreated with BAPTA-AM for $30 \mathrm{sec}$ to buffer intracellular $\mathrm{Ca}^{2+}$ in addition to using hypertonic sucrose stimulation in the presence of EGTA (1 $\mathrm{mm} ; n=5)$. Neither the extent of FM2-10 destaining nor the peak and plateau phase of neurotransmission induced by sucrose was altered under these conditions, suggesting a form of vesicle recycling operating independent of intracellular $\mathrm{Ca}^{2+}$.

$\mathrm{Ca}^{2+}$-dependent high-potassium stimulation $\left(90 \mathrm{~mm} \mathrm{~K}{ }^{+} / 2 \mathrm{~mm}\right.$ $\mathrm{Ca}^{2+}$ ) typically destains up to $60 \%$ of the total fluorescence within $15 \mathrm{sec}$ by mobilizing a larger fraction of the reserve pool (Klingauf et al., 1998). This form of stimulation evoked an electrical response pattern similar to hypertonic stimulation, although the plateau level of transmission reached after the initial decline was $\sim 10 \%$ of the peak regardless of the maturity of synapses (Fig. $1 C, D ; n=6$ each). In experiments using $90 \mathrm{mM} \mathrm{K}^{+}$stimulation, accurate determination of the synaptic responses required subtraction of the inward current induced by high-potassium perfusion. This was achieved by measuring the inward current during $90 \mathrm{~mm} \mathrm{~K}^{+}$application in the presence of postsynaptic glutamate receptor blockers (Fig. 1C, inset). In contrast, electrical responses induced by sucrose application could be fully blocked by receptor inhibitors (data not shown).

In a subset of experiments, we induced neurotransmitter release with extracellular stimulation to trigger action potentials at a frequency of $30 \mathrm{~Hz}$ (Fig. 2). The time constant of synaptic depression was significantly slower in mature synapses (>10 div; $n=8)$ compared with synapses at $6-7$ div cultures $(n=9)$. In the case of mature synapses, responses declined with a time constant of $4.7 \mathrm{sec}$, whereas immature synapses showed a rapid depression with a time constant of $2.7 \mathrm{sec}$. This developmental slowdown in the rate of depression is presumably attributable to a decrease in release probability that was observed previously in other experimental settings (Bolshakov and Siegelbaum, 1995; Choi and Lovinger, 1997; Pouzat and Hestrin, 1997). Despite differences in the rate at which the plateau level of transmission is reached between immature and mature synapses, the relative size of the sustained transmission at $30 \mathrm{sec}$ after the onset of stimulation was not significantly different ( $\sim 25 \%$ for each case). Taken together, these observations indicate the persistence of a substantial level of synaptic transmission under intense sustained stimulation.
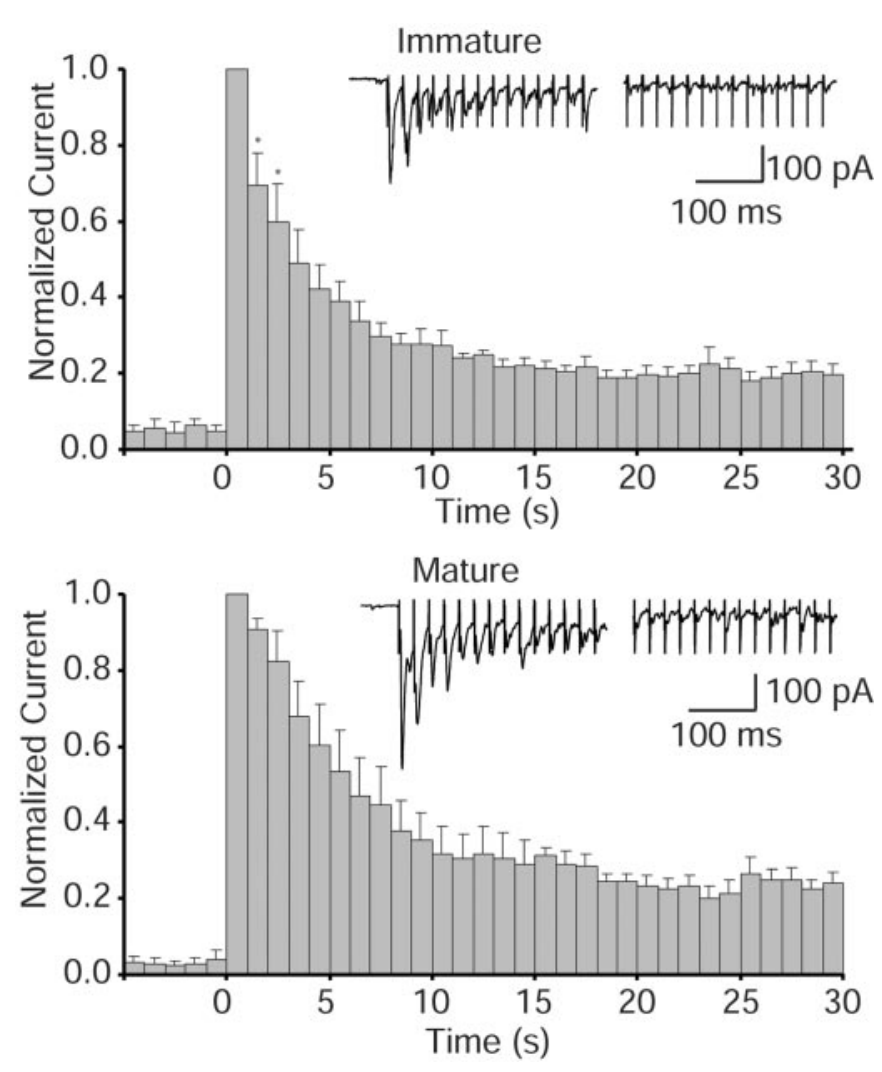

Figure 2. Time course of synaptic responses to sustained $30 \mathrm{~Hz}$ stimulation. The synaptic activity evoked by the $30 \mathrm{~Hz}$ field stimulation $(30 \mathrm{sec})$ was monitored in immature (top; $n=9$ ) and mature (bottom; $n=8$ ) cultures. Young synapses displayed faster depression kinetics in the first 2 sec of stimulation compared with the mature synapses. *Statistical difference at $p<0.05$. Each bar of the graph represents total current integrated over $1 \mathrm{sec}$ intervals of field stimulation and normalized with respect to the maximum. Insets, Evoked synaptic currents by the first and last 14 pulses during $30 \mathrm{sec}$ application of $30 \mathrm{~Hz}$ stimulation.

\section{Morphological correlates of vesicle depletion in response to $90 \mathrm{~mm} \mathrm{~K} / 2 \mathrm{~mm} \mathrm{Ca}^{2+}$ stimulation}

To determine the extent of physical synaptic vesicle depletion that can be induced with prolonged intense stimulation, we analyzed electron micrographs obtained from cultures at 8 div stimulated with $90 \mathrm{mM} \mathrm{K}^{+} / 2 \mathrm{mM} \mathrm{Ca}^{2+}$ stimulation for $10 \mathrm{~min}$ and immediately fixed with glutaraldehyde (Fig. 3). The choice of this paradigm was prompted by our observation that among the stimulation protocols we tested previously, $90 \mathrm{mM} \mathrm{K}^{+} / 2 \mathrm{mM} \mathrm{Ca}^{2+}$ resulted in the most substantial synaptic depression. When compared with control samples incubated in $4 \mathrm{mM} \mathrm{K}^{+} / 2 \mathrm{mM} \mathrm{Ca}^{2+}$ for $10 \mathrm{~min}$, high-potassium stimulation resulted in a modest decrease in the total number of synaptic vesicles (control, $24.4 \pm 20.3$; $n=63$ boutons; after $90 \mathrm{mM} \mathrm{K}{ }^{+}, 15.8 \pm 14.4 ; n=87$ boutons; mean $\pm \mathrm{SD})$. We also observed several membranous structures presumably of endocytic or endosomal origin in $90 \mathrm{mM} \mathrm{K}^{+}$-treated samples (Takei et al., 1996; Richards et al., 2000) (Fig. 3B). In the electron micrographs studied, a significant number of synapses contained synaptic vesicle clusters located near the plasma membrane, although they did not appear to possess docked vesicles. After $90 \mathrm{~mm} \mathrm{~K}^{+}$treatment, there was a small reduction in the number of these synapses $(38 \%$ in control vs $33 \%$ after $90 \mathrm{~mm}$ $\mathrm{K}^{+}$). In other boutons, which had morphologically docked vesicles, there was some decrease in the number of docked vesicles (control, $4.4 \pm 1.5$ vs $3.2 \pm 1.5$ after $90 \mathrm{mM} \mathrm{K}^{+}$). Relatively 
A
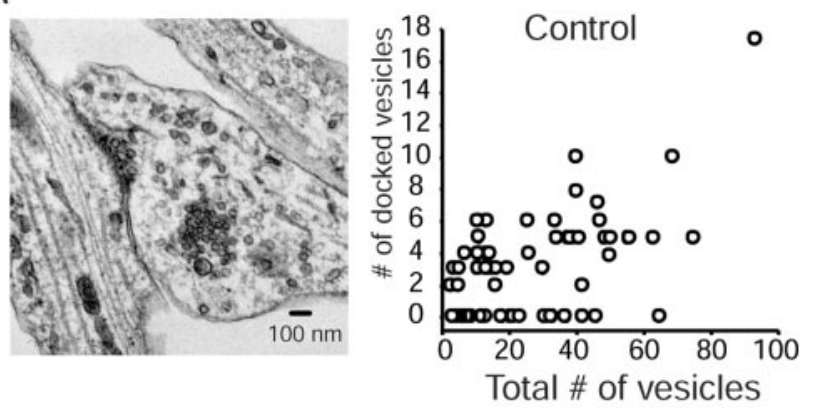

B
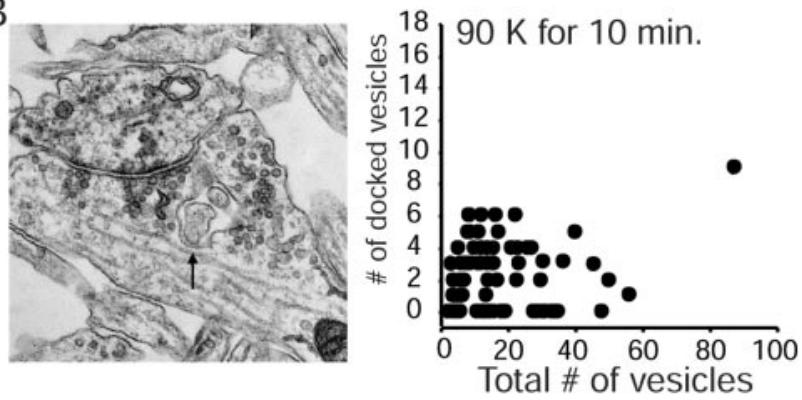

Figure 3. Prolonged high- $\mathrm{K}^{+}$stimulation caused minimal changes in the numbers of vesicles and synapse morphology. $A, B$, Example electronmicrographs illustrate the vesicle organization of synapses from cultures treated with $4 \mathrm{mM} \mathrm{K}^{+}(A)$ and $90 \mathrm{mM} \mathrm{K}^{+}(B)$ for $10 \mathrm{~min}$. Right panels, Cumulative data plotted as the number of morphologically docked vesicles versus the total number of vesicles in synapses treated with $4 \mathrm{mM} \mathrm{K}^{+}$ ( $n=63$ boutons) or $90 \mathrm{mM} \mathrm{K}^{+}$for $10 \mathrm{~min}(n=87$ boutons). The arrow in $B$ points to one of the endosomal structures that were abundant after prolonged $90 \mathrm{mM} \mathrm{K}^{+}$stimulation.

moderate alterations in the organization of vesicles in synapses after intense stimulation argues for a significant role of vesicle recycling to retain vesicle reavailability and structural homeostasis of synapses.

\section{Synapses retain their fast recycling capability after prolonged intense stimulation}

The relative structural integrity of synaptic boutons after prolonged $90 \mathrm{~mm} \mathrm{~K}^{+}$stimulation, with minimal loss of synaptic vesicles, led us to postulate the involvement of a vesicle-recycling mechanism that remains intact in fatigued synapses during intense stimulation. To determine the time course of this recycling process, we performed experiments in which we first challenged synapses with 5 min of uninterrupted $90 \mathrm{~mm} \mathrm{~K}^{+}$stimulation and perfused the styryl dye FM2-10 $(400 \mu \mathrm{M})$ for the last $10 \mathrm{sec}$, followed by fast washout of the extracellular dye and $90 \mathrm{mM} \mathrm{K}^{+}$ solution (Fig. 4A). The choice of FM2-10 was prompted by its fast membrane dissociation kinetics that results in minimal dye retention in vesicles or membrane infoldings that form after intense stimulation (Klingauf et al., 1998; Richards et al., 2000). Destaining induced after a delay of $5 \mathrm{~min}$ was used to assess the extent of loading achieved during this brief application of FM2-10. At the end of each experiment, we determined the size of the total recycling pool by measuring maximal dye loading and destaining. By comparing the two loading levels, we could determine that 10 sec application of FM2-10 stained $\sim 15 \%$ of the total recycling pool (Fig. $4 B, C ; n=5 ; 271$ boutons).

To estimate the time required for reavailability of vesicles endocytosed in the last $10 \mathrm{sec}$ of prolonged $90 \mathrm{~mm} \mathrm{~K}^{+}$stimulation, we reapplied $90 \mathrm{~mm} \mathrm{~K}^{+}$solution for $10 \mathrm{sec}$ with a delay of 5 ,
A
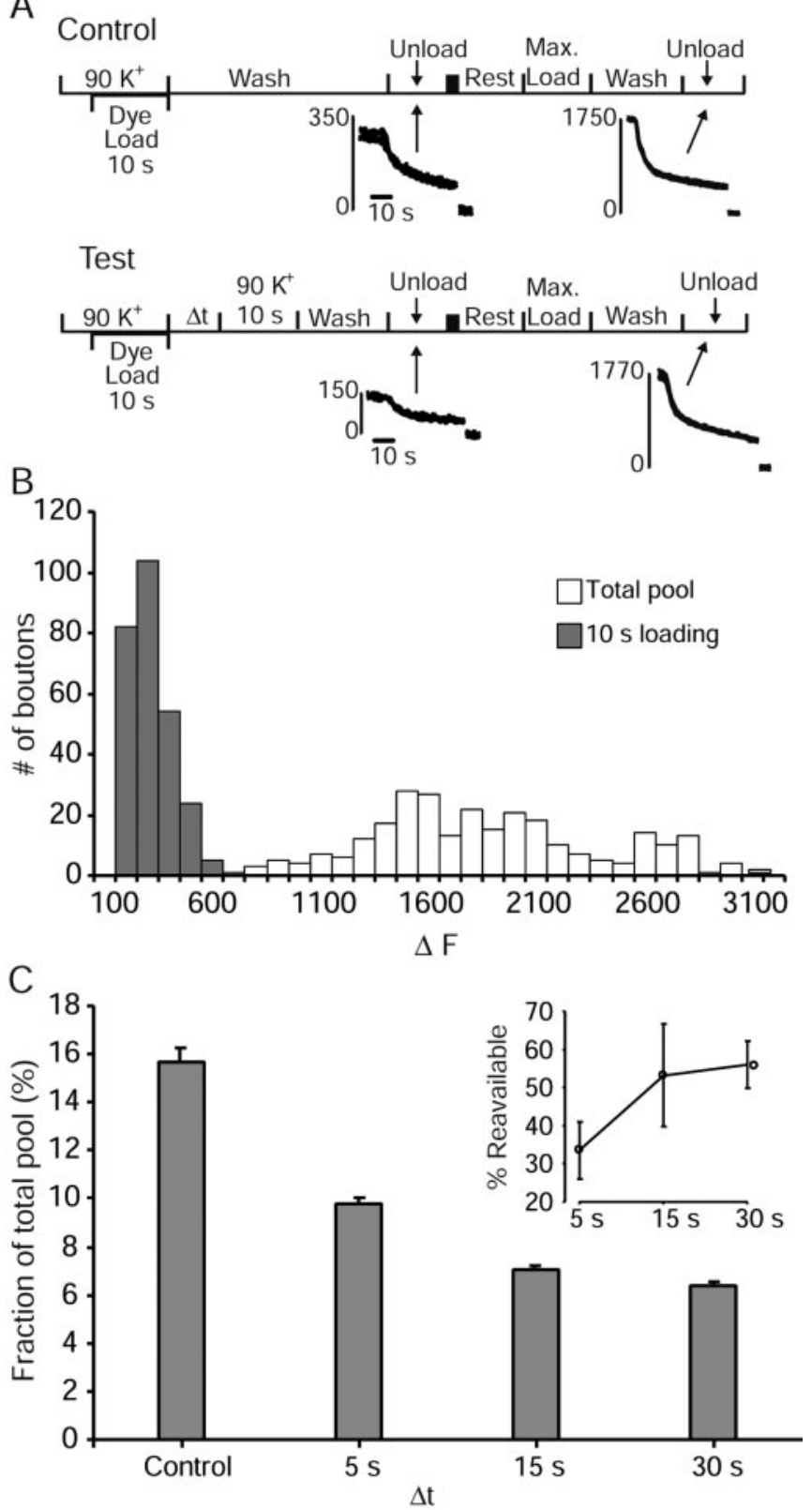

Figure 4. Time course of vesicle recycling during sustained high- $\mathrm{K}^{+}$ stimulation. $A$, Synapses treated with $90 \mathrm{mM} \mathrm{K}^{+}$for 5 min were loaded with FM 2-10 during the last $10 \mathrm{sec}$ of this challenge. The extent of loading achieved by this protocol was determined by measuring the amount of dye unloaded with repeated $90 \mathrm{~mm} \mathrm{~K}^{+}$application after 5 min of washout ( $n=5 ; 271$ boutons). To determine the time course of vesicle reavailability, a brief $(10 \mathrm{sec}) 90 \mathrm{~mm} \mathrm{~K}^{+}$challenge was interspersed after a delay $(\Delta t)$ after dye loading. In three different sets of experiments, a 90 $\mathrm{mM} \mathrm{K}^{+}$test was applied with a delay of $5 \mathrm{sec}(n=7 ; 368$ boutons $), 15 \mathrm{sec}$ ( $n=4 ; 271$ boutons), or $30 \mathrm{sec}(n=4 ; 213$ boutons), respectively. At the end of the washout period, each trial was evaluated for the remaining FM2-10 staining. After each experiment, we executed a maximal dye loading and unloading paradigm to determine the total pool size and normalize the data according to this value. $B$, Histogram of fluorescence intensity distributions in fatigued synapses labeled with the $10 \mathrm{sec}$ staining protocol and total pool size as determined by maximal FM2-10 staining in controls. $C$, After $90 \mathrm{mM} \mathrm{K}^{+}$application for $5 \mathrm{~min}$, up to $15 \%$ of total pool could be stained with FM2-10 (Control). Brief interspersed high-K ${ }^{+}$ challenges partially unloaded the synapses. This fluorescence decrease was first detected at $5 \mathrm{sec}$ after loading and decreased further with increasing delay indicating that more recycled vesicles became available for release. Inset, Percentage of vesicles reavailable with respect to the delay after initial dye uptake (i.e., the difference between fluorescence detected after 5,15 , and $30 \mathrm{sec}$ stimulations and control). 
15 , and $30 \mathrm{sec}$ after rapid washout of FM2-10 (Fig. 4A). If a fraction of the stained vesicles was available for exocy tosis during this period, then it should release the dye in response to this 10 $\sec 90 \mathrm{mM} \mathrm{K}^{+}$stimulation. Furthermore, the extent of destaining achieved by this interspersed stimulation should increase as vesicles become reavailable. More than $30 \%$ of the vesicles that were stained with FM2-10 could be unloaded with a second pulse of 90 $\mathrm{mM} \mathrm{K}^{+}$after a $5 \mathrm{sec}$ delay (Fig. $4 C ; n=7 ; 368$ boutons). When the interval was increased to $15 \sec (n=4 ; 271$ boutons) and 30 sec ( $n=4 ; 213$ boutons), the fraction of vesicles reavailable was saturated at $\sim 50 \%$. This indicates that half of the endocytosed vesicles were recycled through a slower route (Fig. $4 C$ ).

The interpretation of these experiments heavily relies on the efficiency of the fast washout of extracellular dye after the initial loading step. Washout was achieved by a fast perfusion system (10 $\mathrm{ml} / \mathrm{min}$ ), and because of the fast membrane-departitioning time constant of FM2-10 (0.6 sec; Klingauf et al., 1998), extracellular dye concentration decreases substantially within seconds. If some residual FM2-10 was still present to stain synapses during trials with the $5 \mathrm{sec}$ delay, this would make our estimation of $\sim 30 \%$ reavailability at this time point an underestimate.

\section{Estimation of the kinetics of vesicle reavailability after onset of intense stimulation}

Results in the previous section show that hippocampal synapses are capable of vesicle recycling at a fast time scale even after 5 min of intense high- $\mathrm{K}^{+}$stimulation. The next set of experiments was designed to complement this estimate by determining the earliest time point when exocytosed vesicles become reavailable after the onset of stimulation. To address this question, we examined the kinetic difference between the rate of FM dye destaining and the time course of neurotransmitter release from a set of hippocampal synapses. The rationale behind these experiments stems from previous observations that during stimulation, FM2-10 can be cleared out of a fused vesicle within $1 \mathrm{sec}$ by departitioning into solution (Ryan et al., 1996; Klingauf et al., 1998; Pyle et al., 2000) or within milliseconds by lateral diffusion in the neuronal membrane (Zenisek et al., 2000). Both of these time frames are faster than the rate of fusion pore closure and endocytic retrieval (Klingauf et al., 1998; Sankaranarayanan and Ryan, 2001). Therefore, recycled vesicles would not contain significant amounts of FM2-10 that could be detected as additional destaining, whereas the same vesicles would be refilled with neurotransmitter after endocytosis that could give rise to additional synaptic responses. This difference between the two reporters of synaptic vesicle mobilization should result in a deviation between the kinetics of FM2-10 destaining and neurotransmitter release at a point at which recycled vesicles start to be reused.

Figure 5 illustrates the procedure we used to analyze these experiments. In this particular experiment, we evoked neurotransmitter release by application of hypertonic sucrose solution onto a pyramidal cell (Fig. $5 A$ ). In the same region, we simultaneously recorded fluorescent destaining kinetics from synapses that were formed on the dendrites of the same cell (Fig. 5B). Although we have observed a reasonable correlation between the number of visually selected synapses and the size of electrical synaptic responses, we cannot exclude potential minor disparities between the two populations of synapses. Hypertonic sucrose application was sustained for at least $25 \mathrm{sec}$ until destaining reached a plateau. In mature synapses, this level corresponded to $30 \%$ of the total pool as determined by multiple applications of high-K ${ }^{+}$stimulation after a 1 min rest period (Pyle et al., 2000).
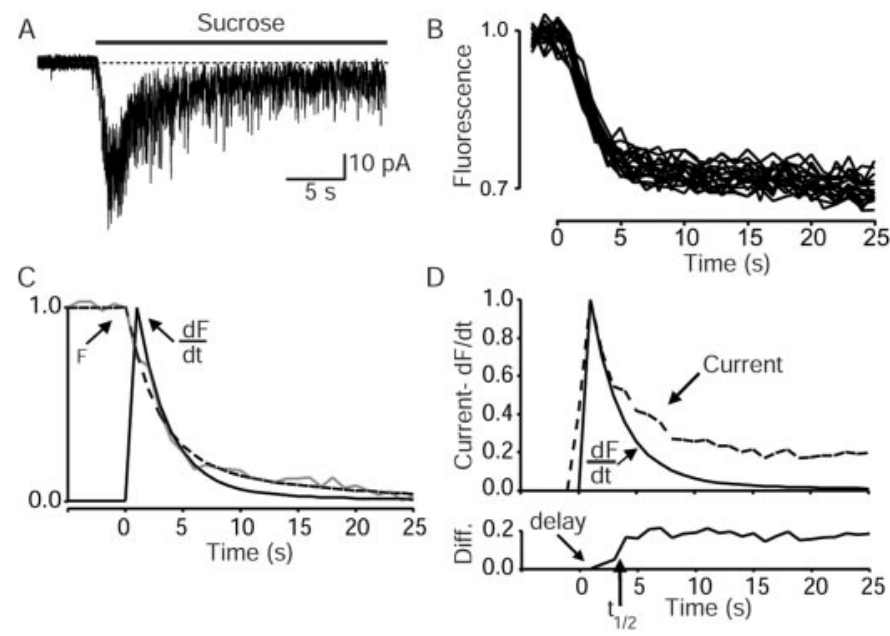

Figure 5. Evaluation of sucrose-induced release by simultaneous electrical recordings and fluorescence destaining. $A, B$, Whole-cell electrical recording of a sucrose response $(A)$ and its instantaneous fluorescence counterpart from multiple boutons on the same neuron $(B) . C$, Average fluorescence response ( $F$, gray line) was smoothed by curve fitting (dashed line) to reduce noise, which in turn helped obtain a smooth derivative of the fluorescence signal $(d F / d t$, solid line $) . D$, The difference between the rate of dye release and synaptic activity was assessed after alignment of the $d F / d t$ and Current plots with respect to their peaks. Current plot was obtained by integrating current within $1 \mathrm{sec}$ intervals. The difference shown in the bottom graph was interpreted as the time course of vesicle reuse.

Destaining profiles originating from all boutons were averaged and smoothed by fitting with multiple exponential functions (typically four). The derivative of the smoothed destaining profile was calculated to obtain the time-dependent change in the rate of destaining (Fig. 5C,D, dF/dt plot). A smoothing operation was necessitated by the fact that straight differentiation substantially increased signal noise and made comparison with the current signal difficult.

To be able to correlate electrophysiological data at the same resolution with the rate of $\mathrm{FM}$ destaining acquired at $1 \mathrm{~Hz}$, we calculated the total current during synaptic activity over $1 \mathrm{sec}$ intervals and normalized with respect to the maximum (Fig. 5D, Current plot). Then we aligned the normalized $\mathrm{dF} / \mathrm{dt}$ and current plots with respect to the maximum points of the two curves (Fig. $5 D)$. In all experiments analyzed, there was significant agreement between the time courses and maxima of the two curves. Occasional mismatches in alignment were not larger than $1 \mathrm{sec}$ (Fig. $5 D)$. The result of this analysis revealed a marked divergence between the rate of FM2-10 destaining and neurotransmitter release. After subtraction of the two curves, the difference was interpreted as the time course of recycled vesicles to join neurotransmission (Fig. 5D, bottom panel). We followed the same procedure to estimate the time course of vesicle recycling in response to action potentials induced by extracellular field stimulation.

\section{Conservation of fast recycling during synaptic development}

When we applied this analysis to experiments performed on synapses at distinct stages of maturation, we observed a consistent divergence between the rate of FM2-10 destaining and neurotransmitter release (Fig. 6). At 6-7 div, when synapses initially assemble a readily releasable pool (Mozhayeva et al., 2002), the difference between the two curves emerged within seconds and 
A
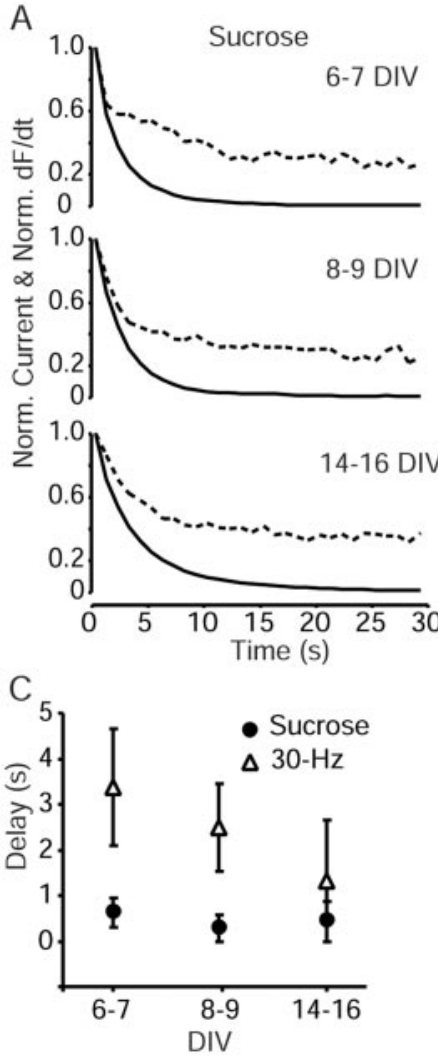
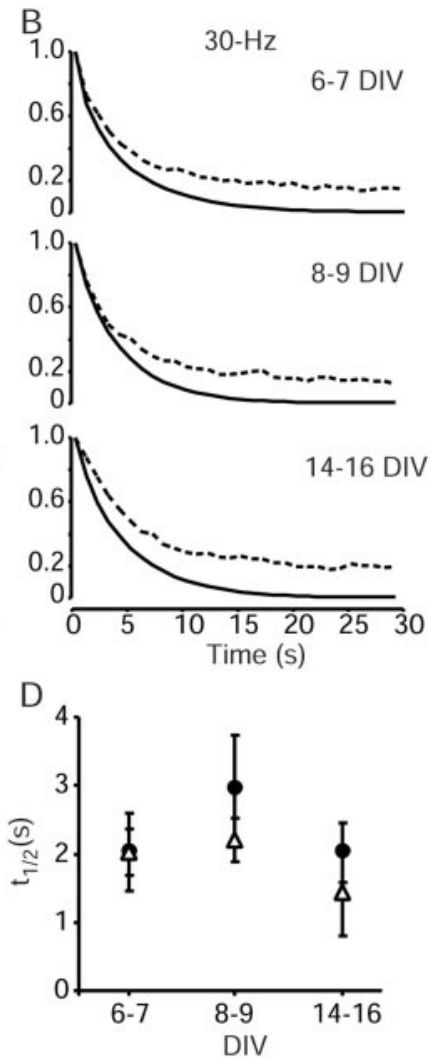

Figure 6. Conservation of fast vesicle recycling during synapse maturation. $A, B$, Comparison of kinetics of dye loss and electrophysiological recordings show that the plateau level of neurotransmitter release cannot be accounted for by styryl dye destaining. This difference between the two modes of measurement was present throughout synapse maturation in response sucrose $(A)$ as well as $30 \mathrm{~Hz}$ stimulation $(B)$. $C$, Average time delay before the initial appearance of the difference between two signals during sucrose (closed circles) and $30 \mathrm{~Hz}$ stimulation (open triangles). D, Rise times $\left(t_{1 / 2}\right)$ of this difference varied between 2 and 3 sec during in vitro development [30 Hz stimulation, $n=9$ (6-7 div), $n=9$ (8-9 div), $n=8$ (14-16 div); sucrose, $n=8$ (6-7 div), $n=8$ (8-9 div), $n=9$ (14-16 div)]. All symbols denote mean values \pm SEM.

reached the maximal level with a $t_{1 / 2}$ of $2-3 \mathrm{sec}$ in response to sucrose stimulation (Fig. $6 A$ ). In the case of $30 \mathrm{~Hz}$ field stimulation, there was larger variability in the delay at which the difference between the $\mathrm{dF} / \mathrm{dt}$ and current plots became significant. In these experiments, both time courses followed each other for longer periods, and their difference was $\sim 30-40 \%$ smaller than in hypertonic sucrose (Fig. 6B). Interestingly, experiments at later stages of maturation at 8-9 and 14-16 div showed a similar divergence of $\mathrm{dF} / \mathrm{dt}$ and current plots, supporting the presence of a fast recycling scheme that enables exocytosed vesicle to rejoin neurotransmission within seconds. The only noticeable tendency during synapse maturation was observed in $30 \mathrm{~Hz}$ experiments in which the delay at which the vesicles contributed to neurotransmitter release became progressively shorter with development (Fig. 6C). This result may suggest an increase in the efficiency of $\mathrm{Ca}^{2+}$-dependent regulation of the vesicle-recycling machinery during synapse maturation.

Morphological and functional studies estimate that the readily releasable pool of hippocampal synapses contain up to 10 vesicles (Schikorski and Stevens, 1997; for review, see Südhof, 2000). In our experiments, the vesicles released within the first few seconds of stimulation with $30 \mathrm{~Hz}$ or sucrose correspond to the RRP

(Rosenmund and Stevens, 1996; Pyle et al., 2000). The kinetic difference between the rate of FM2-10 destaining and neurotransmitter release indicates that most of these vesicles are reavailable within $5 \sec \left(t_{1 / 2} \sim 2-3 \mathrm{sec}\right)$ after the onset of stimulation (Fig. $6 D)$. This time frame therefore suggests a rate of vesicle reuse of up to two vesicles per second, indicating a contribution of recycled vesicles to neurotransmission within seconds after the onset of stimulation.

\section{Estimation of the extent of vesicle pool turnover during stimulation}

An alternative to the analysis presented above involves comparison of the integral of neurotransmitter release to FM2-10 destaining. This approach was used previously by Betz and Bewick (1993) to examine vesicle recycling in the frog neuromuscular junction. In contrast to differentiation, integration does not increase noise of the current signal; thus it does not require smoothing. Therefore, this operation avoids potential errors associated with the smoothing and differentiation of FM2-10 traces. However, one caveat of the integration method is the difficulty in proper alignment and scaling of the electrical and optical signals. To circumvent this problem, first we used the assumption that both electrophysiological events and FM dye destaining originate from the same pool of vesicles released within $30 \mathrm{sec}$ of stimulation, disregarding the potential contribution of recycled vesicles. When we aligned the cumulative integral of electrophysiological responses and FM-destaining patterns from mature synapses (8-16 div) using this assumption, we detected a paradoxical mismatch between the two curves, which suggested FM dye destaining in the absence of glutamate release (Fig. 7A, $B$, open circles). Because FM dye-loaded vesicles were resident within the recycling pool for at least $10 \mathrm{~min}$ before stimulation, all of these vesicles would also be expected to contain neurotransmitter. This contradiction could be alleviated once we modified the earlier assumption and presumed that the vesicle pool detected by electrophysiology is larger than the pool detected by FM dye destaining because of fast vesicle recycling. To incorporate this assumption into our calculations, we scaled the fluorescence trace until it was aligned with the integrated current trace for the first $2 \mathrm{sec}$, our previous estimate for the delay in vesicle reavailability. In the case of hypertonic sucrose stimulation, FM2-10 destaining could account for only $40 \%$ of the electrically detected neurotransmitter release (Fig. $7 A$, filled circles). During $30 \mathrm{~Hz}$ stimulation, on the other hand, dye release comprised $80 \%$ of the electrical signal (Fig. $7 B$, filled circles). This difference may arise from the fact that $30 \mathrm{~Hz}$ stimulation releases up to $60 \%$ of the total pool within first $30 \mathrm{sec}$, whereas hypertonic sucrose stimulation is typically restricted to $20-$ $30 \%$ of the total recycling pool (Pyle et al., 2000; Y. Sara and E. T. Kavalali, unpublished observations).

\section{Rate of vesicle reuse during $10 \mathrm{~Hz}$ stimulation}

Evidence from previous studies at hippocampal synapses has suggested a slower rate of endocytosis during weak stimulation at frequencies between 1 and $10 \mathrm{~Hz}$. This difference is attributed to the effect of intracellular $\mathrm{Ca}^{2+}$ on the regulation of endocytic machinery (Ryan et al., 1996; Klingauf et al., 1998; Kavalali et al., 1999b; Sankaranarayanan and Ryan, 2001). To test the impact of a slower endocytic rate on vesicle reavailability, we measured the rate of FM2-10 destaining and synaptic depression at $10 \mathrm{~Hz}$ stimulation ( $n=10$ experiments; 431 boutons total). During 90 sec of stimulation, fluorescence decreased to $20 \%$ of the initial 

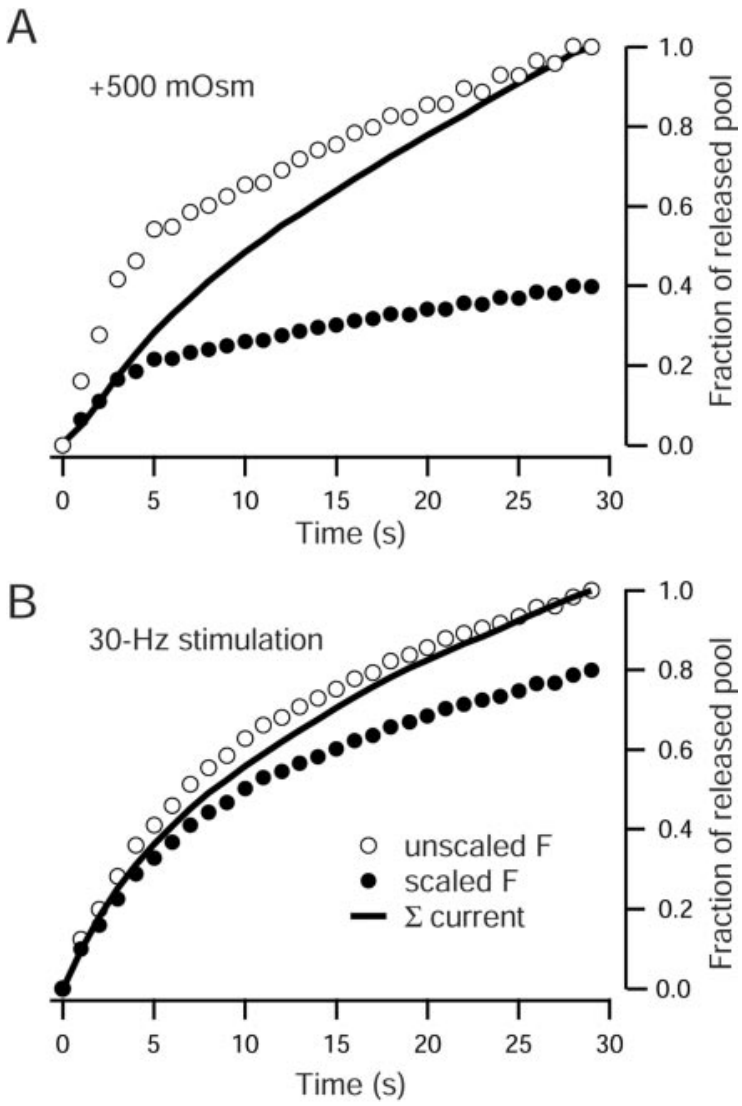

Figure 7. Estimation of the extent of vesicle pool turnover during stimulation. $A, B$, Comparison of the cumulative integral of synaptic current (dark line) to kinetics of fluorescence loss from FM2-10-loaded synapses in mature cultures (8-16 div). Open circles represent average destaining kinetics scaled with the assumption that both electrophysiological and optical readout of exocytosis originate from the same pool of vesicles. Note the apparent mismatch between the curves. When the fluorescence trace was scaled to fit the first $2 \mathrm{sec}$ of neurotransmitter release, the resulting curve (closed circles) revealed a significant difference between the extent of vesicle pool mobilization and neurotransmitter release. The same analysis was performed for hypertonic sucrose stimulation $(A)$ and $30 \mathrm{~Hz}$ field stimulation $(B)$. The relative amount of vesicle reuse is larger during hypertonic sucrose stimulation. However, it should be noted that $30 \mathrm{~Hz}$ stimulation overall mobilizes a larger percentage of the total pool compared with sucrose.

level (Fig. $8 A$ ). In contrast, during $30 \mathrm{~Hz}$ stimulation $(n=4 ; 281$ boutons), fluorescence rapidly decreased to $40 \%$ of the initial level, followed by a slow decline to $30 \%$. Overall, $10 \mathrm{~Hz}$ stimulation mobilizes a significantly larger fraction of the total pool within the first $90 \mathrm{sec}$. Electrical responses, on the other hand, reach a plateau phase of $\sim 20-30 \%$ of the initial peak (Fig. $8 B$ ). To estimate the rate of vesicle reavailability under $10 \mathrm{~Hz}$ stimulation, we applied the analysis described in Figure 5 to synapses stimulated at this frequency. Alignment of average $\mathrm{dF} / \mathrm{dt}$ and current plots revealed a small divergence in kinetics beginning between 5 and $10 \mathrm{sec}$ after onset of stimulation (Fig. $8 B$ ). The divergence between the two curves increased significantly after the initial $20 \mathrm{sec}$. This result indicates a slower rate at which exocytosed vesicles are reused for neurotransmission during 10 $\mathrm{Hz}$ stimulation, presumably because of limitations imposed by the rate of endocytic retrieval and vesicle mobilization at this frequency.

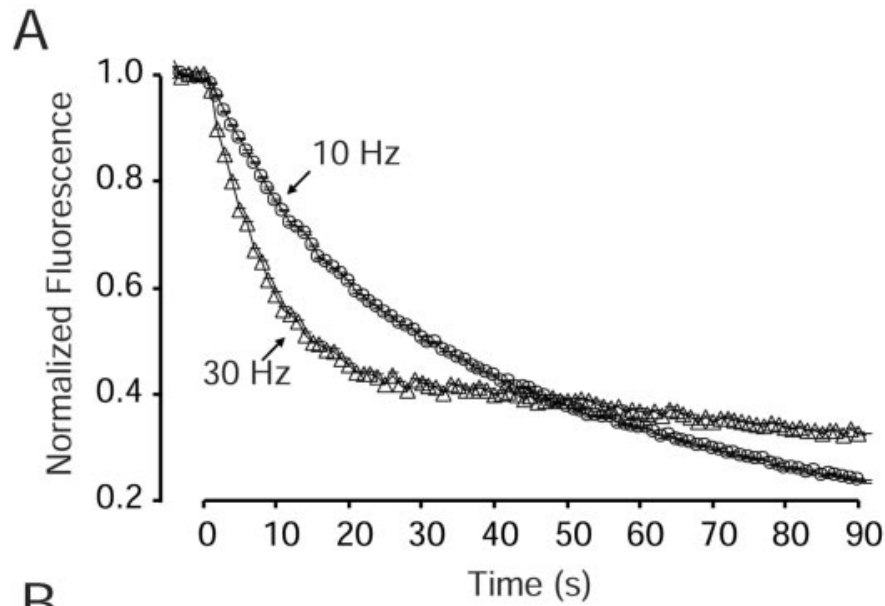

B
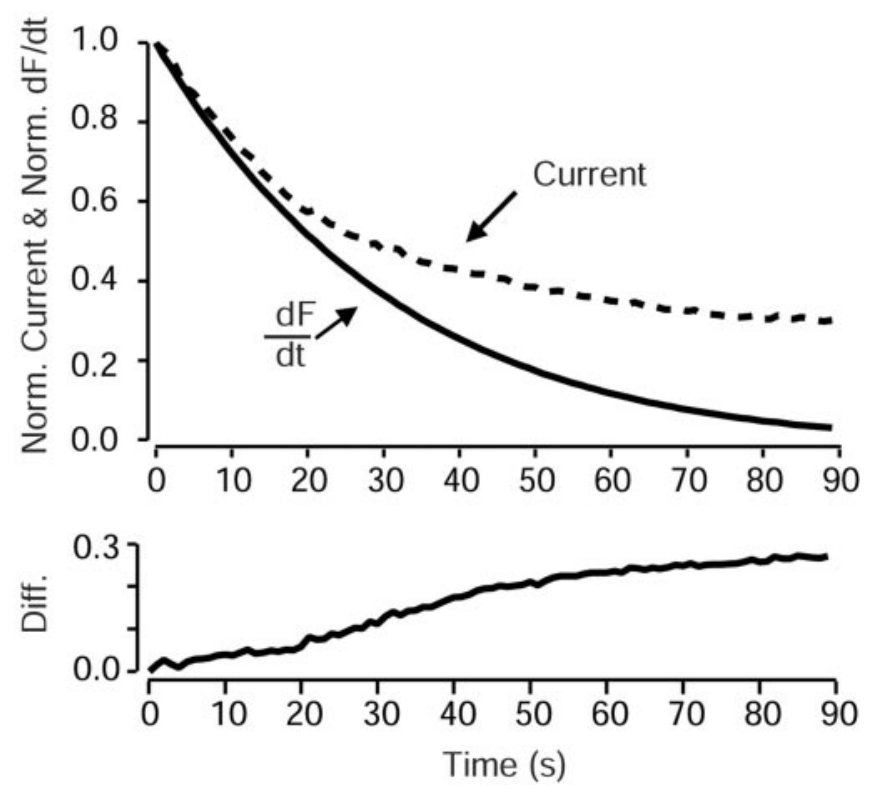

Figure 8. Vesicle mobilization during $10 \mathrm{~Hz}$ stimulation. $A$, In contrast to the biphasic nature of fluorescence loss during $30 \mathrm{~Hz}$ stimulation $(n=$ $4 ; 288$ boutons), in which a fast drop of fluorescence was accompanied by a slow decline, $10 \mathrm{~Hz}$ resulted in monophasic fluorescence loss $(n=6 ; 431$ boutons). The two destaining patterns crossed each other after $50 \mathrm{sec}$. $B$, Analysis of average release kinetics acquired through whole-cell recordings $(n=10)$ and styryl dye destaining induced by $10 \mathrm{~Hz}$ stimulation. Analysis was performed as described in Figure 5. The difference between $d F / d t$ and Current traces became gradually more significant after $20 \mathrm{sec}$ of stimulation. After this point, transmitter release reached to a plateau level, whereas dye release continued to decrease. Bottom graph, Difference between the two traces indicating the time course of vesicle reuse.

\section{Relationship between the sustained phase of synaptic activity and kinetics of vesicle reuse}

To test whether the kinetic parameters for vesicle reuse (estimated in the previous section) can account for the sustained phase of synaptic activity during depression, we set up a kinetic model (Fig. 9A). We restricted this model to the simplest case of sucrose stimulation in which there was minimal mobilization of vesicles from the reserve pool as evidenced by the limited destaining achieved even after prolonged application. Therefore, initially we placed all vesicles in a single compartment corresponding to the readily releasable pool $\left(\mathrm{C}_{0}\right)$. After sucrose application, vesicles in compartment $\mathrm{C}_{0}$ could be mobilized to the fused state $\mathrm{C}_{1}$. We set 
A

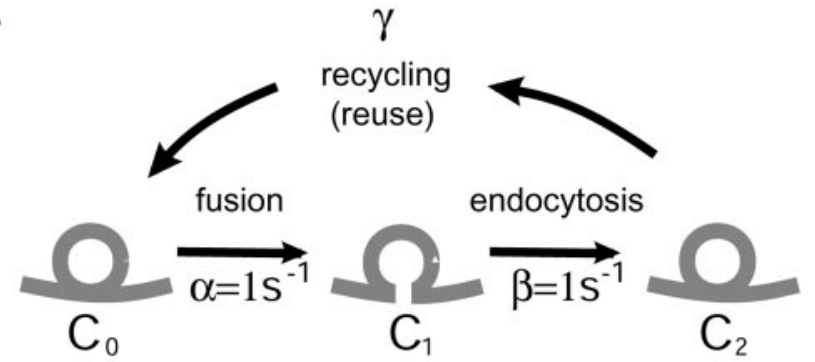

B

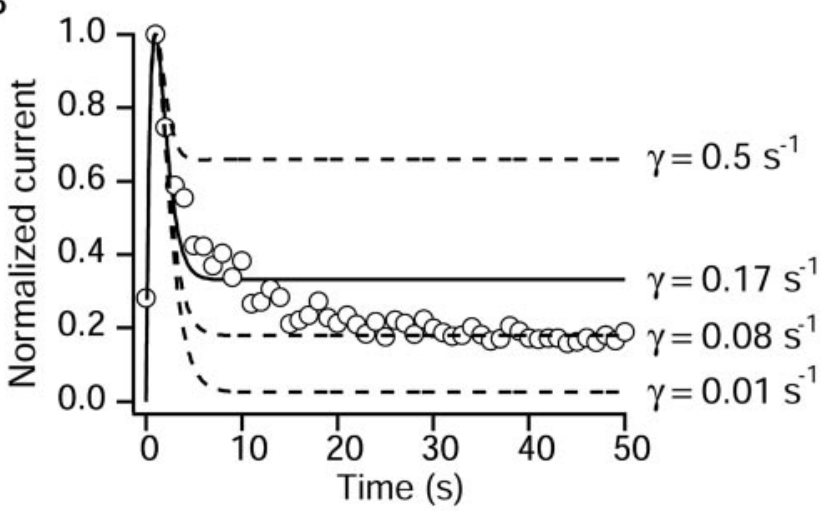

C

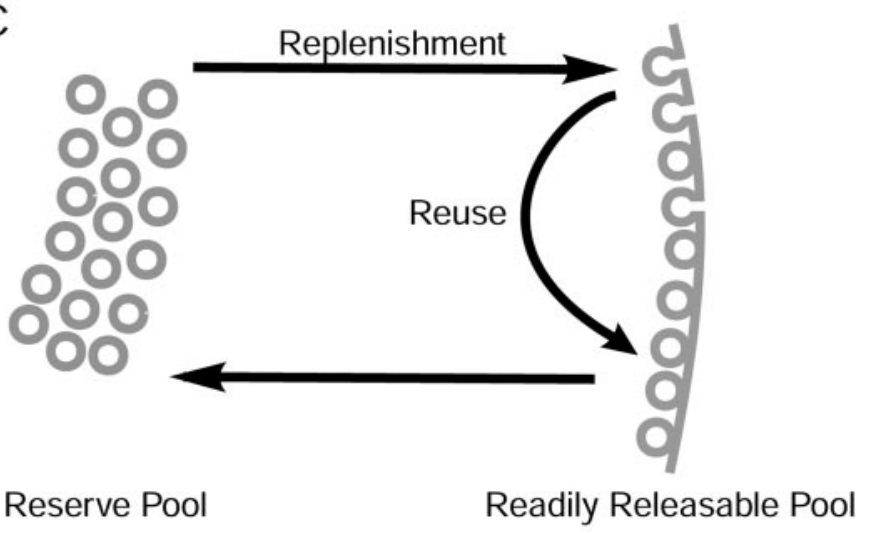

Figure 9. The estimated time frame of vesicle reuse can account for the sustained phase of synaptic activity. $A$, Three-compartmental model where vesicles sequentially move between compartments $C_{0}, C_{1}$, and $C_{2}$ connected with rate constants $\alpha, \beta$, and $\gamma$, respectively. The observable output of the model is detected as the fused state $C_{1}$ by simultaneous solution of the equations: $d C_{0} / d t=-\alpha C_{0}+\gamma C_{2}, d C_{1} / d t=\alpha C_{0}-\beta C_{1}$, and $d C_{2} / d t=\beta C_{1}-\gamma C_{2}$ where $\alpha=\beta=1 /$ sec. $B$, Correspondence between results of the simulation described in $A$ (lines) and average data obtained from prolonged sucrose applications (open circles). Solid line, Time course of vesicles moving through the fused state $\left(C_{1}\right)$ when $\gamma=$ $0.17 / \mathrm{sec}\left(\tau_{\text {reuse }}=5.8 \mathrm{sec}\right)$, which effectively describes the first $15 \mathrm{sec}$ of sucrose response. Dashed lines, Estimations for distinct values of $\gamma \cdot \gamma=$ $0.08 / \mathrm{sec}$ can account for the response at the end of sucrose application. $C$, Simple scheme depicting the organization of functionally distinct vesicle pools and the routes of vesicle replenishment and reuse.

this rate $(\alpha)$ at $1 / \mathrm{sec}$, because this period fairly describes the onset of synaptic responses on sucrose application. The rate of endocytosis, or the rate of movement from $\mathrm{C}_{1}$ to $\mathrm{C}_{2}$, on fusion $(\beta)$ was set at $1 / \mathrm{sec}$ according to previous estimate of Pyle et al. (2000) under the same stimulation condition. The only variable parameter was the rate vesicle reuse $(\gamma)$ through which vesicles become reavailable for release after endocytosis. When compared with the average data obtained from prolonged hypertonic sucrose applications depicted in Figure 1, the initial phase of the plateau up to $15 \mathrm{sec}$ after onset of stimulation could be well described by vesicle reuse with a time constant between 5 and $6 \sec (\gamma=$ $0.17 / \mathrm{sec}$ ), in close agreement with our estimates from experiments described above (Fig. 9B). However after this initial phase, the plateau level declined to $\sim 20 \%$ of the peak, which could be accounted for with a vesicle reuse time constant of $12.5 \sec (\gamma=$ $0.08 / \mathrm{sec}$ ). This number is in closer proximity to the time constant of vesicle reavailability obtained at the end of prolonged high-K $\mathrm{K}^{+}$ stimulation, suggesting a gradual relaxation in the rate of vesicle reuse during sustained stimulation (Fig. 4C). According to the same model, a vesicle-recycling time constant of $\sim 100 \mathrm{sec}$ did not result in an appreciable plateau phase of activity after initial depression, as depicted in Figure $9 B$. It is interesting to note that this simple kinetic model can account for our estimates under both sucrose and prolonged high- $\mathrm{K}^{+}$application without invoking any necessity to recruit more vesicles than the ones initially mobilized at onset. In the case of sucrose, this pool seems to be restricted to the RRP, whereas high- $\mathrm{K}^{+}$stimulation mobilizes a larger population of vesicles comparable with the total recycling pool.

\section{DISCUSSION}

\section{Contribution of vesicle reuse to neurotransmission during sustained stimulation}

In this study, we evaluated the role of fast vesicle recycling in sustaining neurotransmitter release during early and late phases of synaptic depression. These experiments revealed a substantial contribution of recycled vesicles to neurotransmission induced by hypertonic sucrose, action potentials evoked at $30 \mathrm{~Hz}$ and $90 \mathrm{~mm}$ $\mathrm{K}^{+}$stimulation. A stimulation paradigm as extreme as $90 \mathrm{~mm}$ $\mathrm{K}^{+} / 2 \mathrm{mM} \mathrm{Ca}^{2+}$ for 5 min could generate neurotransmitter release continuously with minimal physical loss of synaptic vesicles. In these experiments, the presence of a sustained phase of neurotransmission without a significant decline in amplitude argued for an equilibrium between vesicle reuse and replenishment from a reserve pool. To determine the contribution of fast vesicle reuse to this equilibrium, we measured the amount of dye uptake at a point 5 min after the onset of stimulation. Up to $30 \%$ of the vesicles endocytosed in a $10 \mathrm{sec}$ time frame were reused within 5 sec, supporting a substantial contribution of vesicle reuse to transmission at this stage. Overall, up to $50 \%$ of the vesicles endocytosed during this plateau phase were reavailable within 30 sec; the rest of these vesicles were presumably recycled through a slower pathway.

To quantify the earliest time point at which recycled vesicles are reused during sustained stimulation, we compared simultaneous optical measurements of vesicle mobilization and electrical detection of neurotransmitter release. Destaining kinetics of FM2-10 provides a one-time marker of exocytosis, because a large fraction $(>80 \%)$ of the intravesicular dye leaves the vesicles even during conditions that favor fast endocytosis ( $\sim 1 \mathrm{sec})$, whereas filling of synaptic vesicles with neurotransmitter can rapidly replenish electrical response after endocytosis. Thus discrepancies between the two modes of measurement provide information on the time frame of vesicle recycling during stimulation. We observed a significant deviation between the derivative of the average fluorescence destaining and the time course of electrical responses. The difference emerged at the onset of stimulation, indicating the presence of a fast recycling process in which exocytosed vesicles are available for rerelease within 1-3 
sec. Interestingly, the time course of this reuse pathway was conserved throughout synapse maturation, bringing functional capacity of immature synapses with small vesicle pools in line with mature presynaptic boutons (Fig. 9C).

A parsimonious interpretation of these experiments, in terms of vesicle reuse, depends on the assumption that both optical and electrical signals are originating from the same population of vesicles. To ensure the validity of this assumption, we loaded synapses with FM2-10 using high-potassium stimulation, which gives rise to a maximal amount of fluorescent labeling in hippocampal synapses (see Materials and Methods). This loading protocol increases the likelihood that all recycling vesicles contained dye before their initial fusion. Our observations may also be explained by delayed release of a vesicle population followed by a "kiss and run" type of endocytosis on the order of milliseconds, thus releasing neurotransmitter without dye destaining (Stevens and Williams, 2000). We consider this possibility unlikely, because we observed a significant match between the amount of dye released during $30 \mathrm{sec}$ application of hypertonic sucrose and the extent of dye loading achieved by the same stimulation (M. Mozhayeva and E. T. Kavalali, unpublished observations). Styryl dye loading into synaptic vesicles is less sensitive to fast endocytosis, because these dyes partition into membranes on the order of milliseconds (Neves and Lagnado, 1999; Pyle et al., 2000).

Among the stimulation paradigms used in this study, the longterm stability of synaptic responses to hypertonicity was the most intriguing. It is difficult to reconcile these responses with a purely mechanical action of osmotic shrinkage (Kashani et al., 2001). This rather nonphysiological stimulation seems to reveal continuous recycling capability of an isolated subset of vesicles, presumably the readily releasable pool, without significant exchange with the reserve pool in the absence of $\mathrm{Ca}^{2+}$. Elevation in baseline intracellular $\mathrm{Ca}^{2+}$ levels during sucrose stimulation did not seem to be involved in this form of recycling, because experiments conducted after preincubation of cells in BAPTA-AM to buffer $\left[\mathrm{Ca}^{2+}\right]_{\mathrm{i}}$ did not prevent the sustained activity. Previous experiments have shown the persistence of sucrose-induced neurotransmitter release after extensive $\mathrm{Ca}^{2+}$ buffering (Rosenmund and Stevens, 1996) but did not address the question of sustained activity during hyperosmotic stimulation. An interesting corollary of this result is the presence of a $\mathrm{Ca}^{2+}$-independent form of endocytosis and vesicle recycling, which may be explained by an increase in propensity of protein-protein interactions attributable to a reduction in synaptic volume under hyperosmotic conditions (Delaney et al., 1991).

\section{Cellular mechanisms that underlie fast vesicle recycling}

After exocytosis, synaptic vesicles are recycled and readied for secondary rounds of release through multiple pathways. A "classical pathway" involves endocytosis outside the active zone and incorporates steps to recover fully collapsed vesicles through formation of clathrin coats and recycling through endosomal intermediates (Heuser and Reese, 1973; Holroyd et al., 1999). Alternatively, recycling vesicles can form from membrane invaginations (which may also develop into "cisternae"-like structures) outside the active zone through clathrin-dependent mechanisms (Takei et al., 1996; Koenig and Ikeda, 1996; Richards et al., 2000). Recent evidence suggests that vesicles may recycle by skipping the endosomal intermediates and retain their identity through the vesicle cycle (Koenig and Ikeda, 1996; Murthy and Stevens, 1998;
Richards et al., 2000). Functional evidence in hippocampal synapses also indicates the presence of a more direct pathway where synaptic vesicles can be reused without ever leaving the active zone, presumably at the original sites of release (Klingauf et al., 1998; Pyle et al., 2000; Stevens and Williams, 2000; Südhof, 2000). This pathway is reminiscent of previous proposals of a kiss and run type of endocytosis by Ceccarelli et al. (1973). Morphological studies in the Drosophila neuromuscular junction revealed a ring of endocytic machinery around active zones. This organization of preassembled machinery would significantly increase the efficiency and speed of pathways without endosomal intermediates (Roos and Kelly, 1999). Hot spots for endocytosis near active zones were also observed in detailed electron microscopic studies in the snake neuromuscular junction (Teng et al., 1999; Teng and Wilkinson, 2000). In contrast to previous work, experiments presented in this study did not rely on the kinetic difference between destaining rates of synapses loaded with the styryl dyes FM1-43 and FM2-10. This kinetic difference was interpreted as an indicator of a kiss and run-like mechanism (Klingauf et al., 1998; Kavalali et al., 1999b). Therefore, our current results solely present timing constraints on fast vesicle recycling without any morphological suggestion for an underlying physical mechanism. Nevertheless, within the time frames demonstrated in these experiments, it would be highly unlikely for vesicles to traverse endosomes.

\section{Critical role of fast vesicle recycling as a functional homeostatic mechanism}

In the frog neuromuscular junction, Betz and Bewick (1993) measured a deviation between the summed amplitudes of endplate potentials and FM1-43 destaining that is detectable at $\sim 90$ sec after stimulation at frequencies between 2 and $30 \mathrm{~Hz}$. This study used an analysis procedure similar to the one outlined in Figure 7, using the assumption that neurotransmitter release and FM dye destaining should line up for the first 30-60 sec of stimulation. Evidently this assumption may result in an oversight of fast recycling emerging at early time points (within seconds). Furthermore, in the neuromuscular junction, FM1-43 labels a large pool of reserve vesicles far exceeding FM2-10 labeling of a fast recycling pool. This difference may skew measurements toward slow recycling. This differential labeling of the two pools with distinct FM dyes may not be as prominent in hippocampal synapses (Pyle et al., 2000; Harata et al., 2001). However, we also cannot exclude the possibility that the difference between the results of Betz and Bewick (1993) and the present study originates from a genuine difference in vesicle use between the two preparations. Hippocampal synapses may use faster recycling pathways to ensure more economical use of their limited vesicle supply. Interestingly, recent experiments in Drosophila neuromuscular junction that compared the rate of synaptic depression between wild type and an endocytosis mutant shibire revealed a recycling rate of one to two vesicles per second per active zone (Delgado et al., 2000). The two vesicles per second estimate for fast recycling we provide here is consistent with these results from the Drosophila neuromuscular junction.

Taken together, our results support a significant role for vesicle recycling and reuse in attainment of neurotransmitter release during sustained stimulation throughout synaptic development. Intense stimulation patterns with robust onset such as $30 \mathrm{~Hz}$ or hypertonic sucrose activate a rapid pathway where released vesicles are preferentially reused for neurotransmitter release within seconds. During $10 \mathrm{~Hz}$ stimulation, however, the rate of vesicle 
reavailability slows down, and a larger portion of the reserve pool seems to be used by conventional physical replenishment of the RRP. Therefore, these results expose the regulation of vesiclerecycling pathways within a presynaptic terminal as a critical functional homeostatic mechanism that ensures the most efficient use of the limited number of recycling vesicles present in a CNS synaptic terminal.

\section{REFERENCES}

Betz WJ, Bewick GS (1993) Optical monitoring of transmitter release and synaptic vesicle recycling at the frog neuromuscular junction. J Physiol (Lond) 460:287-309.

Bolshakov VY, Siegelbaum SA (1995) Regulation of hippocampal transmitter release during development and long-term potentiation. Science 269:1730-1734.

Ceccarelli B, Hurlbut WP, Mauro A (1973) Turnover of transmitter and synaptic vesicles at the frog neuromuscular junction. J Cell Biol 57:499-524.

Choi S, Lovinger DM (1997) Decreased probability of neurotransmitter release underlies striatal long-term depression and postnatal development of corticostriatal synapses. Proc Natl Acad Sci USA 94:2665-2670.

Delaney K, Tank DW, Zucker RS (1991) Presynaptic calcium and serotonin-mediated enhancement of transmitter release at crayfish neuromuscular junction. J Neurosci 11:2631-2643.

Delgado R, Maureira C, Oliva C, Kidokoro Y, Labarca P (2000) Size of vesicle pools, rates of mobilization, and recycling at neuromuscular synapses of a Drosophila mutant, shibire. Neuron 28:941-953.

Fiala JC, Feinberg M, Popov V, Harris KM (1998) Synaptogenesis via dendritic filopodia in developing hippocampal area CA1. J Neurosci 18:8900-8911.

Harata N, Pyle JL, Aravanis AM, Mozhayeva M, Kavalali ET, Tsien RW (2001) Limited number of recycling vesicles in small CNS nerve terminals: implications for neural signaling and cell biology of vesicular cycling. Trends Neurosci 24:637-643.

Heuser JE, Reese TS (1973) Evidence for recycling of synaptic vesicle membrane during transmitter release at the frog neuromuscular junction. J Cell Biol 57:315-344.

Holroyd C, Kistner U, Annaert W, Jahn R (1999) Fusion of endosomes involved in synaptic vesicle recycling. Mol Biol Cell 10:3035-3044.

Kashani AH, Chen BM, Grinnell AD (2001) Hypertonic enhancement of transmitter release from frog motor nerve terminals: $\mathrm{Ca}^{2+}$ independence and role of integrins. J Physiol (Lond) 530:243-252.

Kavalali ET, Klingauf J, Tsien RW (1999a) Activity-dependent regulation of synaptic clustering in a hippocampal culture system. Proc Natl Acad Sci USA 96:12893-12900.

Kavalali ET, Klingauf J, Tsien RW (1999b) Properties of fast endocytosis at hippocampal synapses. Philos Trans R Soc Lond B Biol Sci 354:337-346.

Klingauf J, Kavalali ET, Tsien RW (1998) Kinetics and regulation of fast endocytosis at hippocampal synapses. Nature 394:581-585.

Koenig JH, Ikeda K (1996) Synaptic vesicles have two distinct recycling pathways. J Cell Biol 135:797-808.

Liu G, Tsien RW (1995) Properties of synaptic transmission at single hippocampal synaptic boutons. Nature 375:404-408.

Mozhayeva, M, Kavalali ET (2000) Early stages in establishment of vesicle pool hierarchy in hippocampal synapses. Soc Neurosci Abstr 26:83.

Mozhayeva MG, Sara Y, Liu X, Kavalali ET (2002) Development of vesicle pools during maturation of hippocampal synapses. J Neurosci 22:654-665.
Murthy VN, Stevens CF (1998) Synaptic vesicles retain their identity through the endocytic cycle. Nature 392:497-501.

Murthy VN, Stevens CF (1999) Reversal of synaptic vesicle docking at central synapses. Nat Neurosci 2:503-507.

Neher E (1998) Vesicle pools and $\mathrm{Ca}^{2+}$ microdomains: new tools for understanding their roles in neurotransmitter release. Neuron 20:389-399.

Neves G, Lagnado L (1999) The kinetics of exocytosis and endocytosis in the synaptic terminal of goldfish retinal bipolar cells. J Physiol (Lond) 515:181-202.

Pouzat C, Hestrin S (1997) Developmental regulation of basket/stellate cell $\rightarrow$ Purkinje cell synapses in the cerebellum. J Neurosci 17:9104-9112.

Pyle JL, Kavalali ET, Piedras-Renteria ES, Tsien RW (2000) Rapid reuse of readily releasable pool vesicles at hippocampal synapses. Neuron 28:221-231.

Regehr WG, Stevens CF (2001) Physiology of synaptic transmission and short-term plasticity. In Synapses (Cowan WM, Südhof TC, Stevens CF, eds), pp 135-175. Baltimore: Johns Hopkins UP.

Richards DA, Guatimosim C, Betz WJ (2000) Two endocytic recycling routes selectively fill two vesicle pools in frog motor nerve terminals. Neuron 27:551-559.

Roos J, Kelly RB (1999) The endocytic machinery in nerve terminals surrounds sites of exocytosis. Curr Biol 9:1411-1414.

Rosenmund C, Stevens CF (1996) Definition of the readily releasable pool of vesicles at hippocampal synapses. Neuron 16:1197-1207.

Ryan TA, Reuter H, Wendland B, Schweizer FE, Tsien RW, Smith SJ (1993) The kinetics of synaptic vesicle recycling measured at single presynaptic boutons. Neuron 11:713-724.

Ryan TA, Smith SJ, Reuter H (1996) The timing of synaptic vesicle endocytosis. Proc Natl Acad Sci USA 93:5567-5571.

Sankaranarayanan S, Ryan TA (2001) Calcium accelerates endocytosis of vSNAREs at hippocampal synapses. Nat Neurosci 4:129-136.

Schikorski T, Stevens CF (1997) Quantitative ultrastructural analysis of hippocampal excitatory synapses. J Neurosci 17:5858-5867.

Stevens CF, Tsujimoto T (1995) Estimates for the pool size of releasable quanta at a single central synapse and for the time required to refill the pool. Proc Natl Acad Sci USA 92:846-849.

Stevens CF, Williams JH (2000) "Kiss and run" exocytosis at hippocampal synapses. Proc Natl Acad Sci USA 97:12828-12833.

Südhof TC (2000) The synaptic vesicle cycle revisited. Neuron 28:317-320.

Takei K, Mundigl O, Daniell L, De Camilli P (1996) The synaptic vesicle cycle: a single vesicle budding step involving clathrin and dynamin. J Cell Biol 133:1237-1250.

Teng H, Wilkinson RS (2000) Clathrin-mediated endocytosis near active zones in snake motor boutons. J Neurosci 20:7986-7993.

Teng H, Cole JC, Roberts RL, Wilkinson RS (1999) Endocytic active zones: hot spots for endocytosis in vertebrate neuromuscular terminals. J Neurosci 19:4855-4866.

Vaughn JE (1989) Fine structure of synaptogenesis in the vertebrate central nervous system. Synapse 3:255-285.

Wilkinson RS, Cole JC (2001) Resolving the Heuser-Ceccarelli debate. Trends Neurosci 24:195-197.

Wu LG, Betz WJ (1998) Kinetics of synaptic depression and vesicle recycling after tetanic stimulation of frog motor nerve terminals. Biophys J 74:3003-3009.

Zenisek D, Steyer JA, Almers W (2000) Transport, capture, exocytosis of single synaptic vesicles at active zones Nature 406:849-854.

Zucker RS (1989) Short-term synaptic plasticity. Annu Rev Neurosci $12: 13-31$. 